\title{
ANÁLISIS Y EVOLUCIÓN TEMPORAL DE LAS SECUENCIAS PLUVIOMÉTRICAS SECAS DE LARGA DURACIÓN EN LA REGIÓN DE MURCIA (1864-2015)
}

\author{
Víctor RUIZ ÁLVAREZ1, Francisco BELMONTE SERRATO², \\ Ramón GARCÍA MARÍN² ${ }^{2}$, Marcos RUIZ ÁLVAREZ3 \\ ${ }^{I}$ Doctorando en Geografia de la Escuela Internacional de Doctorado de la Universidad de Murcia . \\ ${ }^{2}$ Departamento en Geografía. Universidad de Murcia. \\ ${ }^{3}$ Masterando en Tecnología, Administración y Gestión del Agua. Universidad de Murcia. \\ victor.ruiz1@um.es, franbel@um.es, ramongm@um.es, marcos.ruiz1@um.es
}

\section{RESUMEN}

En este estudio se ha analizado la evolución temporal de las secuencias pluviométricas secas en el ámbito territorial de la Región de Murcia (sureste de España), durante el periodo temporal 1864-2015. Para la identificación y caracterización de las mismas, se ha utilizado el Índice Estandarizado de Sequía Pluviométrica (IESP). Este índice permite realizar una categorización de distintos niveles de intensidad de sequía. Se han estudiado dos periodos temporales diferentes (1864-2015 y 1929-2015), dado que hasta la primera década del S.XX, únicamente se dispone de los registros de la serie de Murcia ciudad. Para el análisis del periodo 1929-2015 se han utilizado cinco series de precipitación repartidas por toda la geografía murciana. El principal resultado que se ha obtenido es que en la evolución del IESP de la serie de Murcia 1864-2015, se observa una clara intensificación, en la intensidad, frecuencia y duración de las sequías en las últimas décadas, revelándose un claro aumento en los últimos 50 años de las sequías de carácter excepcional.

Palabras clave: sequía, intensidad, Región de Murcia, excepcional

\begin{abstract}
In this study has been analyzed the temporal evolution of dry sequences, in the territorial of the Region of Murcia (southeast of Spain), during the period 1864-2015. For identification and characterization of droughts, it has been used the Standardized Index of Pluviometry Drought (IESP). This index allow do a categorization about different levels of drought intensity. It has been studied two different period, because, it is available only the register of the station of Murcia city, until the first decade of the twentieth century. It has been used five station of rainfall for the period 1929-2015. These station are spread for all territory of Region of Murcia. The main results it has been obtained is that in the evolution of the serie of Murcia (1864-2015), is observed a process of intensification of the droughts in the Region of Murcia in the last fifty years, especially in intensity, frequency and duration of droughts exceptional.
\end{abstract}

Key words: drought, intensity, Region of Murcia, exceptional 


\section{INTRODUCCIÓN}

El estudio y análisis de la evolución de los extremos pluviométricos, como son las sequías e inundaciones, es de vital importancia debido a las enormes repercusiones territoriales y socioeconómicas, que dichos riesgos naturales de origen climático conllevan sobre un determinado espacio geográfico, más si cabe, si tal y como se manifiesta en numerosas investigaciones científicas, como en el Quinto informe de evaluación sobre el cambio climático (AR5,2013), realizado por el Grupo Intergubernamental de Expertos sobre el Cambio Climático (Intergovernamental Panel on Climate Change, IPCC), se está produciendo en las últimas décadas una intensificación de la frecuencia e intensidad en las latitudes medias del Hemisferio Norte $\left(30-60^{\circ} \mathrm{N}\right)$ de dichos fenómenos extremos de origen climático.

En concreto en el AR5, se observa que se ha producido una intensificación en la intensidad, frecuencia y duración de las sequías en el ámbito de las regiones mediterráneas europeas, desde la década de 1950. Aunque, se expone que existe un nivel de confianza bajo que reafirme los resultados de esta tendencia, debido a la falta de observaciones directas, las incertidumbres metodológicas y las desigualdades geográficas. Dentro de un ámbito nacional se han realizado numerosos trabajos de investigación que reafirman los resultados mostrados por el AR5, en cuanto a la intensificación de la intensidad, frecuencia y duración de los periodos secos (Sousa et al., 2011; Hoerling et al., 2012). En Sousa et., (2011) se refleja una intensificación de los periodos de indigencia pluviométrica en la mitad oriental de la Península Ibérica. En Hoerling et al., (2012), se habla de una aumento en la intensidad y frecuencia de las sequías en las regiones mediterráneas europeas a partir de la década de 1970. En estos estudios también se observa un descenso no significativo de las precipitaciones en gran parte de la Península Ibérica desde la segunda mitad del S.XX, incluyendo la mitad oriental.

A nivel de la Región de Murcia también se han estudiado las tendencias de las precipitaciones durante las últimas décadas, así como la posible intensificación de las sequías y el impacto de estas sobre el territorio (García Marín y Conesa García, 2006; García Marín, y Calvo García-Tornel, 2008; Sánchez Toribio et al., 2010). En la Región de Murcia y en general en todo el SE peninsular, estos estudios tienen una enorme trascendencia e importancia debido a la elevada variabilidad interanual que caracteriza el clima de esta región, de modo que se suceden largos periodos secos, con episodios de lluvias breves pero muy intensas, que causan graves inundaciones (Martín- Vide y Olcina Cantos, 2001).

En estos estudios de ámbito regional se habla de la acentuación e intensificación de los periodos secos en el SE peninsular desde mediados del S.XX. García Marín y Calvo García-Tornel (2008), muestran que las rachas secas se manifiestan como uno de los hechos climáticos definidores del ambiente mediterráneo, y su transcendencia es sustancial en el funcionamiento de muchos ecosistemas, al generar fuertes alteraciones en la disponibilidad de agua dentro de un ámbito semiárido.

En García Marín y Conesa García (2006), se expone que desde la segunda mitad del S.XX, la pluviometría de la cuenca del Guadalentín (Región de Murcia, SE de España), ha estado caracterizada por apreciables anomalías, siendo la grave secuencia de sequias e inundaciones padecida la que ha sensibilizado a la opinión pública, ya 
que, estos fenómenos climáticos excepcionales y adversos han causado desolación y ruina sobre extensas zonas en este territorio. En Sánchez- Toribio et al., (2010), se expone que los periodos de sequía en el Valle del Guadalentín (Región de Murcia, SE de España) reducen la superficie cultivada sobre todo en cultivos hortícolas, por la gran demanda hídrica y el costo adicional que supone, disminuyendo por tanto la producción, por lo que se llega a la conclusión que la sequía en este territorio de la Región de Murcia se ve agravada por la demanda evaporativa de los cultivos.

En este trabajo se pretende analizar y estudiar la evolución temporal de las secuencias pluviométricas secas de larga duración en el ámbito territorial de la Región de Murcia (Sureste de España), durante el periodo temporal 1864-2015. Para la identificación y caracterización de las mismas, se ha utilizado el Índice Estandarizado de Sequía Pluviométrica (IESP) que fue implementado y propuesto por Pita López (2000). Este índice ha sido aplicado a las series de precipitación más longevas de la Región de Murcia procedentes de los registros de la Agencia Estatal de Meteorología, siendo la serie más antigua la de Murcia ciudad (1864-2015). Este índice permite realizar una categorización de distintos niveles de intensidad de sequía.

Se han analizado dos periodos temporales diferentes (1864-2015 y 1929-2015) dado que hasta la primera década del S.XX, únicamente se dispone de los registros de la serie de Murcia Ciudad. El principal objetivo de este trabajo es estudiar, si en las últimas décadas se está produciendo una intensificación en la Región de Murcia en algunos de los parámetros que definen las sequías (intensidad, frecuencia y duración), tal y como se manifiesta en numerosos estudios, como el Quinto Informe de Evaluación sobre el Cambio Climático realizado por el IPCC (AR5, 2013).

\section{MÉTODOS}

\section{1. Área de estudio}

El ámbito de estudio de este trabajo es la Región de Murcia, la cual se encuentra localizada en el extremo meridional del suroeste del continente europeo, y dentro de la Península Ibérica, como ya ha sido mencionado, en la zona sureste (Figura 1). El territorio analizado cuenta con una extensión de 11.313 km². El territorio de la Región de Murcia ha sufrido a lo largo de la historia los envites y efectos territoriales adversos de las secuencias pluviométricas de larga duración. Gran parte del territorio analizado se encuentra dominado por un ámbito semiárido siendo la precipitación media anual de $321 \mathrm{~mm}$ para el periodo de referencia 1971-2000 (Garrido Abenza et al., 2014).

En la Región de Murcia, como en el resto de las regiones del ámbito mediterráneo, hay una clara dicotomía entre las condiciones climáticas del interior y de la costa. Este hecho se ve claramente reflejado en la variable de las precipitaciones, ya que se pasa de los casi $600 \mathrm{~mm}$ de media anual en el extremo noroccidental, a los alrededor de $200 \mathrm{~mm}$ de media del extremo suroccidental, siendo esta una de las áreas más secas del continente europeo. En este trabajo se ha aplicado el IESP a estaciones situadas en diferentes ámbitos geográficos y climáticos de la Región de Murcia. 


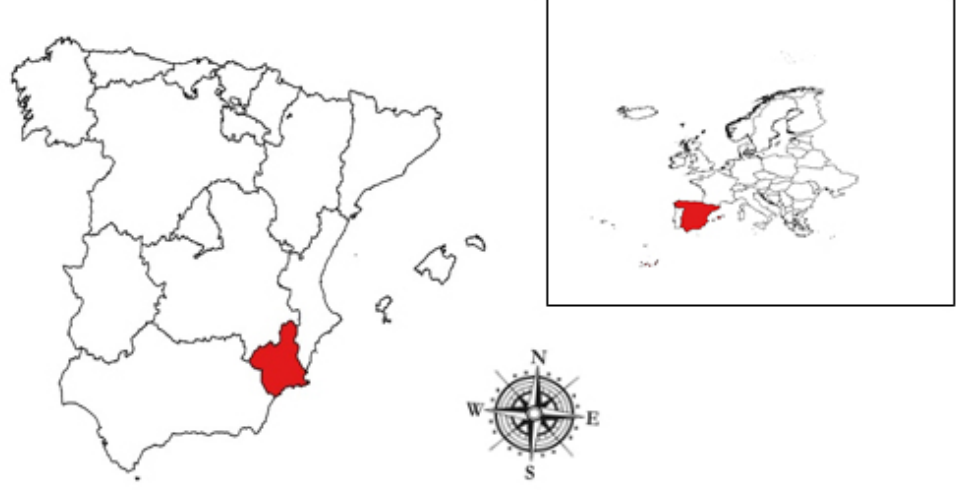

Fig.1: Área de estudio. Elaboración propia

\subsection{Base de datos}

Para la realización de estudio se han utilizado cinco series de precipitación repartidas a lo largo de toda la geografía murciana. En la figura 2 se muestra la distribución espacial de estas cinco estaciones utilizadas y en la tabla 1 la información relativa de las mismas. Las estaciones utilizadas se han extraído de dos bases de datos (Ruiz Álvarez et al., 2014 y Luna et al., 2012) construidas a partir de los registros digitales de la Agencia Estatal de Meteorología. Las series de Murcia-Alcantarilla y Cehegín proceden de la base de datos de Luna et al. (2012).

\begin{tabular}{|ccccccc|}
\hline ID & Nombre & Comienzo & Final & Latitud & Longitud & Altitud $(\mathrm{m})$ \\
\hline $\mathbf{1}$ & CEHEGIN & 1929 & 2015 & & & \\
& CEHEGÍN DOCUMENTOS HISTORICOS & $01-1929$ & $12-1952$ & 38.10 & -1.79 & \\
& 7120 CEHEGIN & $01-1953$ & $12-1986$ & 38.09 & -1.79 & 572 \\
& 7119U CEHEGIN LOS ROSALES & $01-1987$ & $09-2005$ & 38.08 & -1.80 & 580 \\
& 7120C CEHEGÍN CIUDAD & $01-1991$ & $12-2015$ & 38.10 & -1.79 & 563 \\
\hline $\mathbf{2}$ & JUMILLA & 1929 & 2015 & & & \\
& 7138 JUMILLA ESTACION VITICOLA & $01-1929$ & $12-1976$ & 38.47 & -1.33 & 510 \\
& 7138A JUMILLA I L & $01-1977$ & $12-1992$ & 38.47 & -1.36 & 502 \\
& 7138D JUMILLA LA ESTACADA & $01-1993$ & $12-2015$ & 38.46 & -1.34 & 445 \\
\hline $\mathbf{3}$ & TOTANA LA CARRASCA & 1929 & 2015 & & & \\
& 7115 TOTANA LA CARRASCA & $01-1929$ & $12-2015$ & 37.86 & -1.58 & 1200 \\
\hline $\mathbf{4}$ & MURCIA ALCANTARILLA & 1864 & 2015 & & & \\
& 7182C MURCIA INSTITUTO & $01-1864$ & $09-1940$ & 37.98 & -1.13 & 66 \\
\hline & 7228 MURCIA ALCANTARILLA & $10-1940$ & $12-2015$ & 37.96 & -1.23 & 85 \\
\hline
\end{tabular}

Tabla 1: Información básica de las cinco estaciones utilizadas. El ID sirve para identificar cada estación en la figura 2. Elaboración propia 

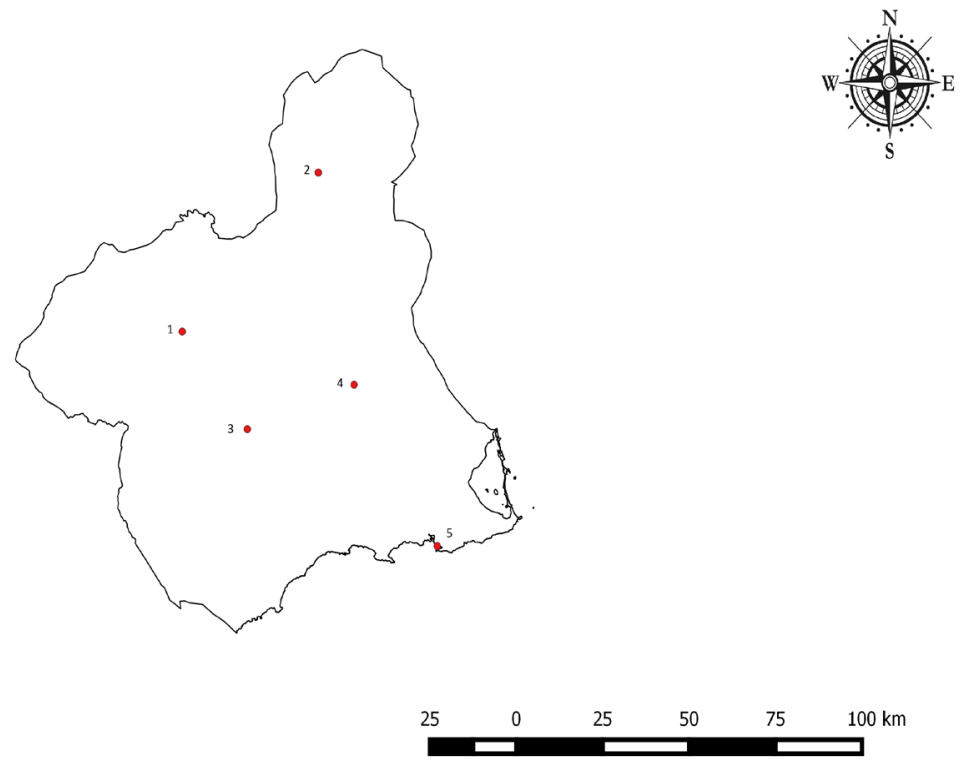

Fig.2: Distribución geográfica de las series de precipitación utilizadas en este trabajo. * Cada número se corresponde con un ID que aparece reflejado en la tabla 1. Elaboración propia

\section{3. Índice Estandarizado de Sequía Pluviométrica (IESP)}

En este estudio, para llevar a cabo la caracterización de las secuencias pluviométricas secas de larga duración, se ha utilizado el Índice Estandarizado de Sequía Pluviométrica (IESP) que fue implementado y propuesto por Pita López (2000), donde se puede consultar la metodología llevada a cabo para su aplicación. Se trata de un índice mensual de sequía meteorológica que utiliza tan solo la variable de las precipitaciones, y está basado en el cálculo de las anomalías pluviométricas mensuales acumuladas y estandarizadas. El IESP es eficaz para identificar las diferentes secuencias secas, ya que permite mostrar con precisión el arranque y el fin de cada sequía, así como su duración e intensidad registrada en cada momento.

El IESP ha sido aplicado a cada una de las 5 series de precipitación utilizadas, y a partir de los valores obtenidos se han establecido una serie de categorías o niveles de sequía (figura 3), en función de los valores de intensidad de sequía (Z) calculados. Las categorías o niveles de sequía que se han obtenido son las siguientes, de menor a mayor intensidad: húmedo, anormalmente seco, sequía moderada, sequía severa, sequía extraordinaria y sequia excepcional.

Los diferentes niveles de sequía se han establecido en función de diferentes probabilidades de excedencia (\%), de determinados valores de intensidad de sequía (Z). Además con la probabilidad de excedencia (\%), se obtienen una serie de periodos de retorno. En este punto, cabe mencionar que la categoría de "sequía excepcional" podría servir para delimitar las sequias excepcionales a las que se alude en la Directiva Marco del Agua (Directiva 2000/60/CE) (figura 3). Para cada una de las series se ha obtenido un determinado umbral para el establecimiento de los diferentes niveles de sequía. 


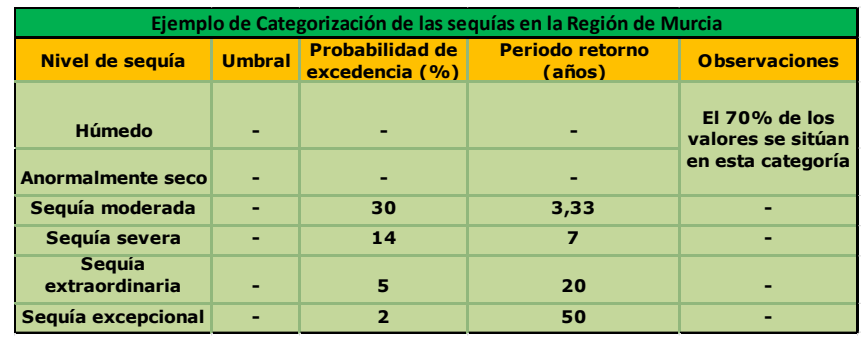

Fig.3: Metodología utilizada por el IESP para la obtención de diferentes niveles de sequía. Elaboración propia

\section{RESULTADOS}

\subsection{IESP de la serie de Murcia-Alcantarilla (1864-2015)}

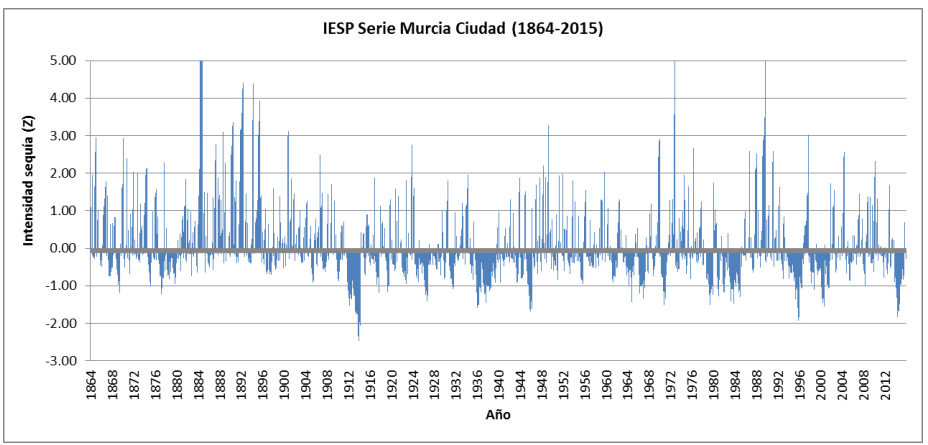

Fig.4: Evolución del IESP de la serie de Murcia-Alcantarilla (1864-2015). Elaboración propia.

\begin{tabular}{|c|c|c|c|}
\hline Periodo & $\begin{array}{c}\text { Duración } \\
\text { (n' }\end{array}$ & $\begin{array}{c}\text { Intessidad } \\
\text { máxima }\end{array}$ & Nivel de sequía \\
\hline JULIO 1874 - JUNIO 1875 & 12 & -1.02 & SEVERA \\
\hline AGOSTO 1876 - AGOSTO 1877 & 13 & -1.23 & EXTRAORDINARIA \\
\hline MARZO 1878 - MARZO 1880 & 24 & -0.95 & SEVERA \\
\hline ABRIL 1911 - ABRIL 1914 & 37 & $-\mathbf{2 . 4 7}$ & EXCEPCIONAL \\
\hline DICIEMBRE 1916 - NOVIEMBRE 1917 & 12 & -1.18 & EXTRAORDINARIA \\
\hline ABRIL 1925 - ENERO 1927 & 22 & -1.41 & EXTRAORDINARIA \\
\hline MARZO 1927-AGOSTO 1928 & 18 & -0.57 & MODERADA \\
\hline AGOSTO 1930 - NOVIEMBRE 1931 & 16 & -1.09 & SEVERA \\
\hline JUNIO 1935 - AGOSTO 1939 & $\mathbf{5 1}$ & -1.59 & EXCEPCIONAL \\
\hline FEBRERO 1940 - FEBRERO 1941 & 13 & -0.92 & SEVERA \\
\hline FEBRERO 1945 - MARZO 1946 & 14 & -1.68 & EXCEPCIONAL \\
\hline NOVIEMBRE 1960 - FEBRERO 1962 & 16 & -0.91 & SEVERA \\
\hline OCTUBRE 1963 - MAYO 1965 & 20 & -1.44 & EXCEPCIONAL \\
\hline ENERO 1966- MAYO 1967 & 17 & -1.34 & EXTRAORDINARIA \\
\hline FEBRERO 1970 - AGOSTO 1971 & 19 & -1.51 & EXCEPCIONAL \\
\hline MAYO 1978 - DICIEMBRE 1979 & 20 & -1.51 & EXCEPCIONAL \\
\hline SEPTIEMBRE 1980 - JULIO 1981 & 11 & -1.28 & EXTRAORDINARIA \\
\hline SEPTIEMBRE 1981 - SEPTIEMBRE 1982 & 13 & -1.19 & EXTRAORDINARIA \\
\hline NOVIEMBRE 1982 - ABRIL 1985 & 30 & -1.48 & EXCEPCIONAL \\
\hline JUNIO 1993 - OCTUBRE 1996 & 41 & -1.93 & EXCEPCIONAL \\
\hline OCTUBRE 1997 - FEBRERO 1999 & 17 & -1.11 & SEVERA \\
\hline ABRIL 1999 - SEPTIEMBRE 2000 & 18 & -1.54 & EXCEPCIONAL \\
\hline NOVIEMBRE 2000 - OCTUBRE 2001 & 12 & -1.08 & SEVERA \\
\hline SEPTIEMBRE 2013 - SEPTIEMBRE 2015 & 24 & -1.84 & EXCEPCIONAL \\
\hline
\end{tabular}

Tabla 3: Periodos de sequía de la serie de Murcia-Alcantarilla (1864-2015). Elaboración propia 


\subsection{IESP de las cinco series más longevas de la Región de Murcia (1929-2015)}

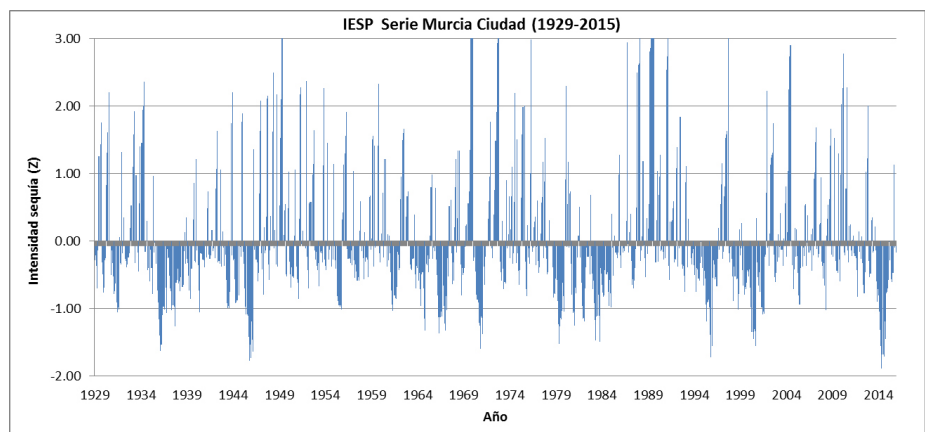

Fig.5: Evolución del IESP de la serie de Murcia-Alcantarilla (1929-2015). Elaboración propia.

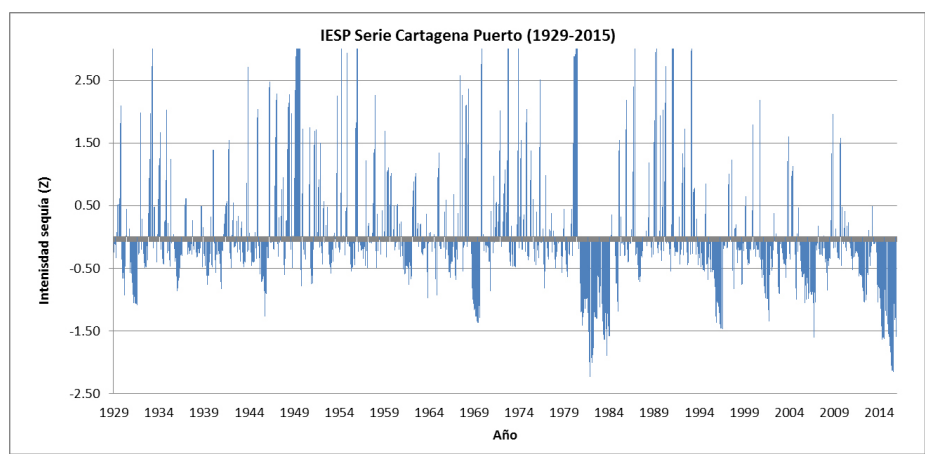

Fig.6: Evolución del IESP de la serie de Cartagena Puerto (1929-2015). Elaboración propia.

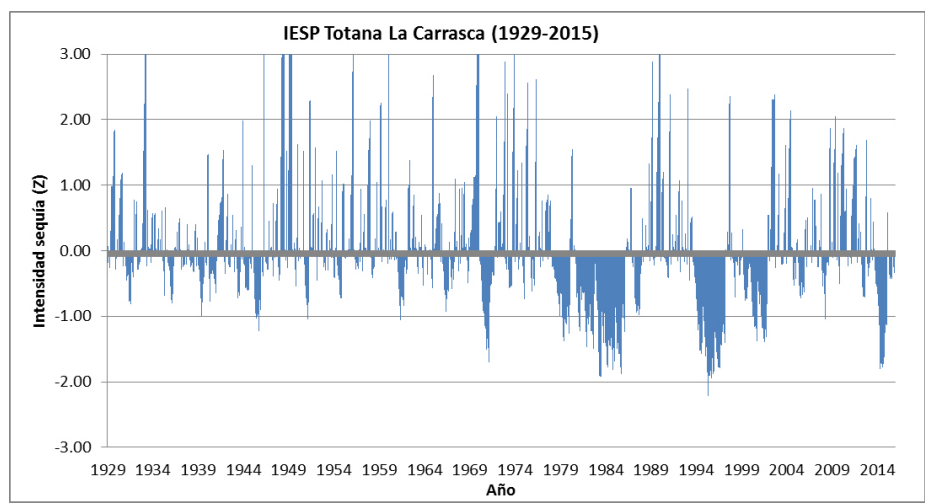

Fig.7: Evolución del IESP de la serie de Totana La Carrasca (1929-2015). Elaboración propia. 


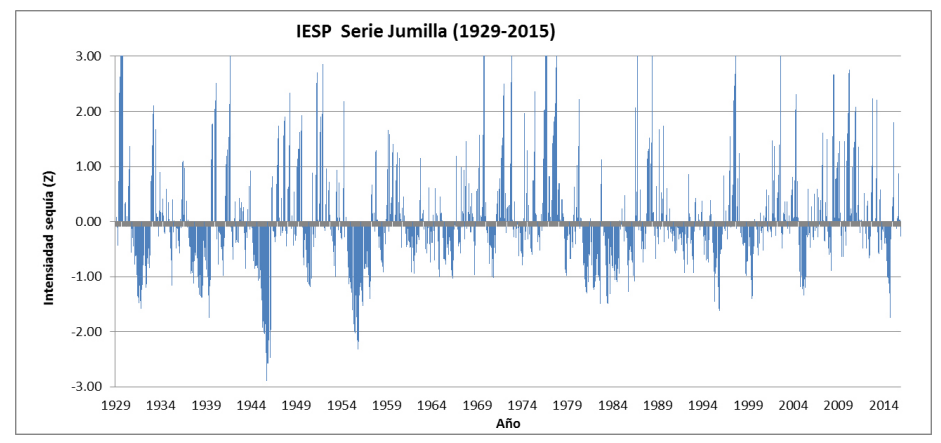

Fig.8: Evolución del IESP de la serie de Jumilla (1929-2015). Elaboración propia.

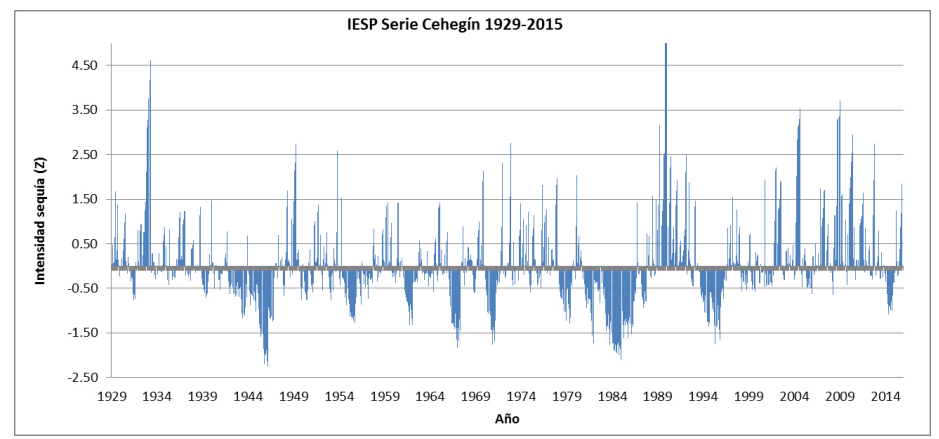

Fig.9: Evolución del IESP de la serie de Cehegín (1929-2015). Elaboración propia.

\section{DISCUSIÓN}

Los resultados obtenidos con la aplicación del IESP a las series más longevas de la Región de Murcia en dos periodos temporales diferentes (1864-2015 y 1929-2015) permiten extraer una serie de conclusiones. El IESP de la serie más longeva utilizada en este estudio (figura 4), la de Murcia-Alcantarilla refleja claramente como se ha producido una intensificación de la frecuencia, intensidad y duración de los periodos de sequía durante la segunda mitad del S.XX, tal y como se apuntado en numerosos estudios anteriormente mencionados en el apartado 1.Introducción.

En la tabla 3 se muestran todos los periodos de sequía registrados en la serie de Murcia-Alcantarilla, que presentan al menos 12 o más meses de duración. En esta tabla se puede observar como durante los 152 años de duración del periodo se han registrado un total de diez sequías de carácter excepcional, que son las que presentan una mayor gravedad por las repercusiones territoriales y socioeconómicas que conllevan, bien por su gran intensidad o por su duración. El hecho más destacable, es que de estas diez sequías excepcionales, siete han tenido lugar en los últimos 50 años y 4 en los últimos 30 años, lo que pone evidencia que se está produciendo un claro aumento en los parámetros de la intensidad y frecuencia de las sequías en la serie más longeva de la Región de Murcia, hecho contrastado en numerosos estudios mencionados en el apartado 1 de este estudio. 
Evidentemente con los datos de una única estación no se pueden extraer resultados concluyentes, y es por ello por lo que se ha aplicado también el IESP a otras cinco series en el periodo 1929-2015 (figuras 5 a 9). En la figura 5 se observa el IESP de la serie Murcia-Alcantarilla pero en este caso en el periodo 1929-2015. A diferencia del periodo 1864-2015 donde se refleja claramente una intensificación de las sequías a partir de la segunda mitad del S.XX, en el periodo 1929-2015 al ser un periodo temporal más corto no se observa tan nítidamente está intensificación de las sequías en las últimas décadas. Esto pone de manifiesto, la importancia del periodo temporal seleccionado, de modo que cuanto más largo sea el mismo mejor se podrán observar las posibles variaciones de los parámetros de las sequías y se podrán obtener mejores resultados.

La evolución del IESP del resto de series longevas de la Región de Murcia (figuras 6 a 9) para el periodo temporal 1929-2015, revela resultados muy dispares. Mientras en el caso de las series de Totana La Carrasca y Cartagena Puerto, se observa un claro aumento en la intensidad, frecuencia y duración de las sequías en los últimos 30 años, las series de Jumilla y Cehegín no revelan dicha intensificación. Por lo tanto, este hecho podría dar lugar a un nuevo estudio, en el que se analizara el comportamiento de las sequías en las comarcas más meridionales de la Región de Murcia (Valle del Guadalentín y Campo de Cartagena), y ver si el comportamiento mostrado por las series de Totana La Carrasca y Cartagena Puerto se extrapola al resto del espacio geográfico de ambas comarcas. Cabe recordar, que estas comarcas son las que recogen una menor cantidad de precipitación anual dentro del ámbito territorial de la Región de Murcia, y que por tanto una posible intensificación de las sequías puede llevar consigo importantes consecuencias socioeconómicas y territoriales.

\section{REFERENCIAS}

- García Marín, R. y Conesa García, C. (2006): Secuencias pluviométricas secas de larga duración en la cuenca del Guadalentín (Murcia- Almería). En: Cuadrat Prats et al. (Eds.): Clima, Sociedad y Medio Ambiente. Publicaciones de la Asociación Española de Climatología (AEC) Serie A, nº5. Zaragoza, pp. 371-382.

- García Marín, R. y Calvo García-Tornel, F. (2008): Frecuencia y evolución de rachas secas en la Cuenca del Guadalentín (Sureste de España). Boletín de la Asociación de Geógrafos Españoles, $n^{\circ} 48$, pp. 71-89.

- Garrido Abenza, R., Palenzuela Cruz, J.E. y Bañon Peregrín, L.M. (2014): Atlas climático de la Región de Murcia. Agencia Estatal de Meteorología. 167 pp.

- Hoerling, M., Eischeid, J., Perlwitz, J., Quan, X.W., Zhang, T., Pegion, P. (2012): On the increased frequency of Mediterranean drought. Journal of Climate, 25, pp. 2146 - 2161. DOI: http://dx.doi.org/10.1175/JCLI-D-11-00296.1

- IPCC (2013): Climate Change 2013: The Physical Science Basis. En Stocker, T.F., Qin, D., Plattner, G.K, Tignor, M., Allen, S.K., Boschung, J., Nauels, A., Xia, Y., Bex., V. and Midgley, P.M. (eds.). Cambridge University Press, Cambridge, United Kingdom and New York, NY, USA.

- Luna, M.Y, Guijarro, J.A. y López, J.A. (2012): Reconstrucción, homogeneidad y tendencias de las series históricas de precipitación mensual acumulada en la España peninsular y las islas baleares. $8^{\circ}$ Congreso Asociación Española de Climatología (AEC), Salamanca, pp. 499-507. 
- Martín-Vide, J. y Olcina Cantos, J. (2001): Climas y tiempos de España. Alianza Editorial. Madrid, $258 \mathrm{pp}$.

- Pita López, M.F. (2000): Un nouvel índice pour les domaines mediterranéens. Application au bassin du Guadalquivir (sud-ouest de l'Espagne). Publications de l'Association Internationale de Climatologie, $\mathrm{n}^{\circ} 13$, pp. 225-233.

- Ruiz Álvarez, V., Sánchez Lorenzo, A. y García Marín, R. (2014): Creación de una base de datos con series largas de precipitación en la Región de Murcia y análisis temporal de la serie media anual, 1914-2013. Revista de Climatología, vol.14, pp. 81-97.

- Sánchez- Toribio, M.I., García Marín, R., Conesa García, C. y López Bermudéz, F. (2010): Evaporite demand and wáter requirements of the principal crops of the Guadalentín valley (SE Spain) in drought periods. Spanish Journal of Agricultural Research, vol.8, pp.66-75. Instituto Nacional de Investigación y Tecnología Agraria y Alimentaria (INIA).

- Sousa, P.M., Trigo, R.M., Aizpurua P., Nieto R, Gimeno L. y Garcia-Herrera, R. (2011): Trends and extremes of drought indices throughout the 20th century in the Mediterranean. Natural Hazards Earth System Sciences, vol.11, pp. 11-33. DOI:10.5194/nhess-11-33-2011 